\title{
Regularly fluctuating somatic cell count pattern in dairy herds
}

\author{
Z. Deng, ${ }^{1 *} \odot$ T. J. G. M. Lam, ${ }^{1,2} \odot$ H. Hogeveen, ${ }^{1,3} \odot$ and G. Koop ${ }^{1} \odot$ \\ ${ }^{1}$ Department Population Health Sciences, Faculty of Veterinary Medicine, Utrecht University, Yalelaan 7, 3584 CL Utrecht, the Netherlands \\ ${ }^{2}$ Royal GD, PO Box 9, 7400 AA Deventer, the Netherlands \\ ${ }^{3}$ Chair Group Business Economics, Wageningen University and Research, PO Box 8130, 6700 EW Wageningen, the Netherlands
}

\begin{abstract}
Online somatic cell count (SCC) measurement is widely used in dairy herds milked with automatic milking systems (AMS) and gives the opportunity to closely monitor individual cow udder health. Using automated SCC data, we observed cows displaying a remarkably regularly fluctuating SCC (rfSCC) pattern, which is described in this study. We aimed to (1) estimate the prevalence of rfSCC in cows milked by AMS, (2) characterize the rfSCC pattern, and (3) identify factors potentially associated with the rfSCC pattern. We analyzed 30-d episodes of composite SCC recordings of 1,000 cows from 55 dairy herds from 6 countries using an AMS with automated SCC measurement, and we identified the rfSCC pattern in $4.7 \%$ (95\% CI: $3.5-6.2 \%$ ) of these episodes. The rfSCC episodes had a median SCC of 701 $\times 1,000$ cells $/ \mathrm{mL}(2.5-97.5 \%$ quantile: $539-1,162)$, a median amplitude of $552 \times 1,000$ cells $/ \mathrm{mL}(2.5-97.5 \%$ quantile: 409-886), and a median cycle length of 4.1 d (2.5-97.5\% quantile: 3.7-4.9). Bacteriological culture data from quarter-milk samples collected every $2 \mathrm{wk}$ in 1 Dutch AMS herd were analyzed, yielding no clear association between pathogen species and the rfSCC pattern found in that herd. Altogether, we described an intriguing phenomenon, present in almost $5 \%$ of the cows during a 1-mo study period. Further work is needed to quantify its importance in terms of udder health, but also to elucidate the mechanism behind this remarkable SCC pattern.
\end{abstract}

Key words: automatic milking system, regularly fluctuating somatic cell count, mastitis

\section{INTRODUCTION}

The SCC is the most often used diagnostic tool in udder health management of dairy cows (Schukken et al., 2003). Typically, SCC is measured by collecting

Received December 21, 2020.

Accepted June 4, 2021.

*Corresponding author: zhaoju.deng2014@gmail.com composite milk during a monthly test and performing a laboratory test, often as part of a DHI approach. In such systems, SCC is measured for all cows in a herd every 4 to 6 wk. Following the development of automatic milking systems (AMS) and the developments in sensor technology, online devices have been constructed to enumerate or estimate SCC automatically (Sørensen et al., 2016; Deng et al., 2020). Such tools allow for highly frequent SCC measurements, as frequently as every milking, enabling the study of the temporal variation in SCC in detail over longer periods of time and in large numbers of animals and herds.

Recently, when we were working with daily SCC measurements, we noticed that several cows shared a similar SCC pattern that we defined as a regularly fluctuating SCC (rfSCC). We described rfSCC as a string of SCC records of a cow that displayed a regular cyclic fluctuating behavior, going up and down within several days. The patterns we observed were often stable and lasted for multiple weeks of time. The cyclicity and stability of the pattern were surprising and suggest that we need a better understanding of this phenomenon.

At present, it is unclear how often rfSCC occurs, what the amplitude and duration of the cyclic pattern is, what causes it, and what other factors are related to the occurrence of rfSCC. An association with IMI seems obvious and needs to be investigated. Thus, the aims of this study were to (1) determine the prevalence of rfSCC, (2) characterize the rfSCC pattern by estimating the amplitude, duration, and median SCC of the rfSCC pattern, and (3) identify factors associated with the occurrence of the rfSCC pattern.

\section{MATERIALS AND METHODS}

In this research, data from 2 observational field studies were analyzed. Data set 1 consisted of routinelycollected recordings of composite milk SCC estimated by online California Mastitis Test (O-CMT) using an interpolation algorithm, which yielded an approximation of the SCC, in 55 AMS herds in 6 countries collected between January 1, 2015, and April 29, 2016, as described previously (Deng et al., 2020). This data 
set was used to characterize the rfSCC pattern and estimate its occurrence. For data set 2, we collected O-CMT data in 1 Dutch AMS herd (about 60 cows) for approximately 6 mo between September 26, 2017, and February 27, 2018, and performed bacteriological culture every 2 wk of quarter-milk samples of each cow in the herd.

\section{Identification of fSCC Patterns and Estimation of the Prevalence}

The raw data of data set 1 consisted of farm identification, cow identification, and the O-CMT measurements accompanied by the timestamp of the milking. The frequency of O-CMT measurements varied between farms between once every third milking up to every milking, dependent on AMS settings. We used O-CMT data of 11,219 lactating cows in 55 AMS herds, which were available for approximately $1 \mathrm{yr}$ (Deng et al., 2020). As a first step in the data preparation, the O-CMT recordings for each cow were cut into episodes of 30 consecutive days, resulting in 201,519 episodes of 11,219 cows. Only episodes with $\geq 1$ O-CMT recordings per day on each of the $30 \mathrm{~d}$ were included, which we considered high frequent O-CMT recording (the overall herd average number of milkings per cow per day was $3.1,2.5-97.5 \%$ quantile: $2.7-3.8$ ), resulting in 9,756 episodes from 2,762 cows on 55 farms. Then, a total of 1,000 cows were randomly selected from these 2,762 cows. Finally, for each selected cow, we randomly selected 1 episode. These 1,000 episodes were displayed as a graph with the milking date and time at the $\mathrm{x}$-axis and the O-CMT reading for each milking at the y-axis. Black dots indicated the O-CMT measurements, which were connected by lines. These graphs had a fixed layout, with the $\mathrm{x}$-axis ranging from $\mathrm{d} 1$ to 30 , and the $\mathrm{y}$-axis ranging from 0 to $5,000,000$ cells $/ \mathrm{mL}$. A dashed horizontal line was added to represent 200,000 cells/ $\mathrm{mL}$. The 4 authors of this paper, all active mastitis researchers, acted as raters and independently classified these 1,000 graphs into 1 of the following 8 categories: (1) continuously regularly fluctuating (the rfSCC pattern of interest), showing a cyclic fluctuation of O-CMT between high and low values and showing a regular fluctuating pattern during the entire episode; (2) partially continuously regularly fluctuating, showing the cyclic fluctuating pattern mentioned under (1) during only part of the episode; (3) irregularly fluctuating, showing fluctuation of O-CMT over time, but not showing a regular pattern; (4) stable high, showing a constantly high O-CMT throughout the episode; (5) stable low, showing a constantly low O-CMT throughout the episode; (6) stable low with $\geq 1$ peak(s), showing a constantly low O-CMT, with 1 or more brief peaks of single high O-CMT measurements; (7) increasing, showing a transition from a low O-CMT to a high OCMT during the episode; (8) decreasing, showing a transition from a high O-CMT to a low O-CMT during the episode. In the descriptions above, low O-CMT was interpreted as up to approximately 200,000 cells/mL and high as above approximately 200,000 cells/mL. A representative example for each category is provided in Figure 1. Before the actual assessment was carried out, 30 test graphs were independently classified by the 4 raters, and discrepancies were discussed afterward. Based on this discussion, the definitions were further adjusted, resulting in the definitions above. The graphs were presented to every rater in a random order and without any further information on the background of these episodes in an online questionnaire. In the questionnaire, the raters could see the O-CMT graphs and a multiple-choice question representing the 8 categories, of which 1 could be checked. The questionnaire can be accessed at https://wj.qq.com/s2/6397417/2176/.

The results of this questionnaire were cross tabulated, and all graphs that were categorized as pattern 1 (continuously regularly fluctuating SCC; $\mathrm{n}=27$ ) or pattern 2 (partially regularly fluctuating $\mathrm{SCC} ; \mathrm{n}=64$ ) by at least 1 of the 4, but not all 4, raters were discussed within the group, leading to a collective judgment for each of these graphs. The proportion of pattern 1 and pattern 2 was calculated as the number of graphs collectively identified as such divided by 1,000 . In addition, we calculated the proportion using the number of episodes with the patterns of interest based on agreement of the initial judgment of all 4 raters (the most conservative estimate) and based on $\geq 1$ of the raters initially choosing this pattern (the most liberal estimate). Interrater agreement of the initial ratings was evaluated by Fleiss kappa, which was calculated using the kappam.fleiss function in the irr package (Gamer, 2019) in $\mathrm{R}$ version 3.6.2 (https://www.r-project.org/). To calculate the contribution of cows with rfSCC pattern to the herd SCC, we first calculated the product of SCC and milk yield for each milking, then we summed up these products for each cow and herd per day. The contribution of cows with rfSCC pattern to the herd SCC was calculated as the cow-level sums of products of SCC and milk yield divided by the herd level sums of products of SCC and milk yield for each day.

\section{Characterization of the rfSCC Pattern}

The episodes from data set 1 that were collectively identified as rfSCC pattern (pattern 1) were characterized by spectral analysis that accounted for the unevenly spaced measurements using the spec.lomb function from the spectral package (Seilmayer, 2019) in 
$\mathrm{R}$ to calculate the duration and amplitude of cycles as well as the average SCC within the 30-d episode. The SCC dynamics of the 22 cows with rfSCC pattern were provided in Supplemental Figures S1 to S22 (https:/ /data.mendeley.com/datasets/rz69vxxm99/1; Deng, 2021).
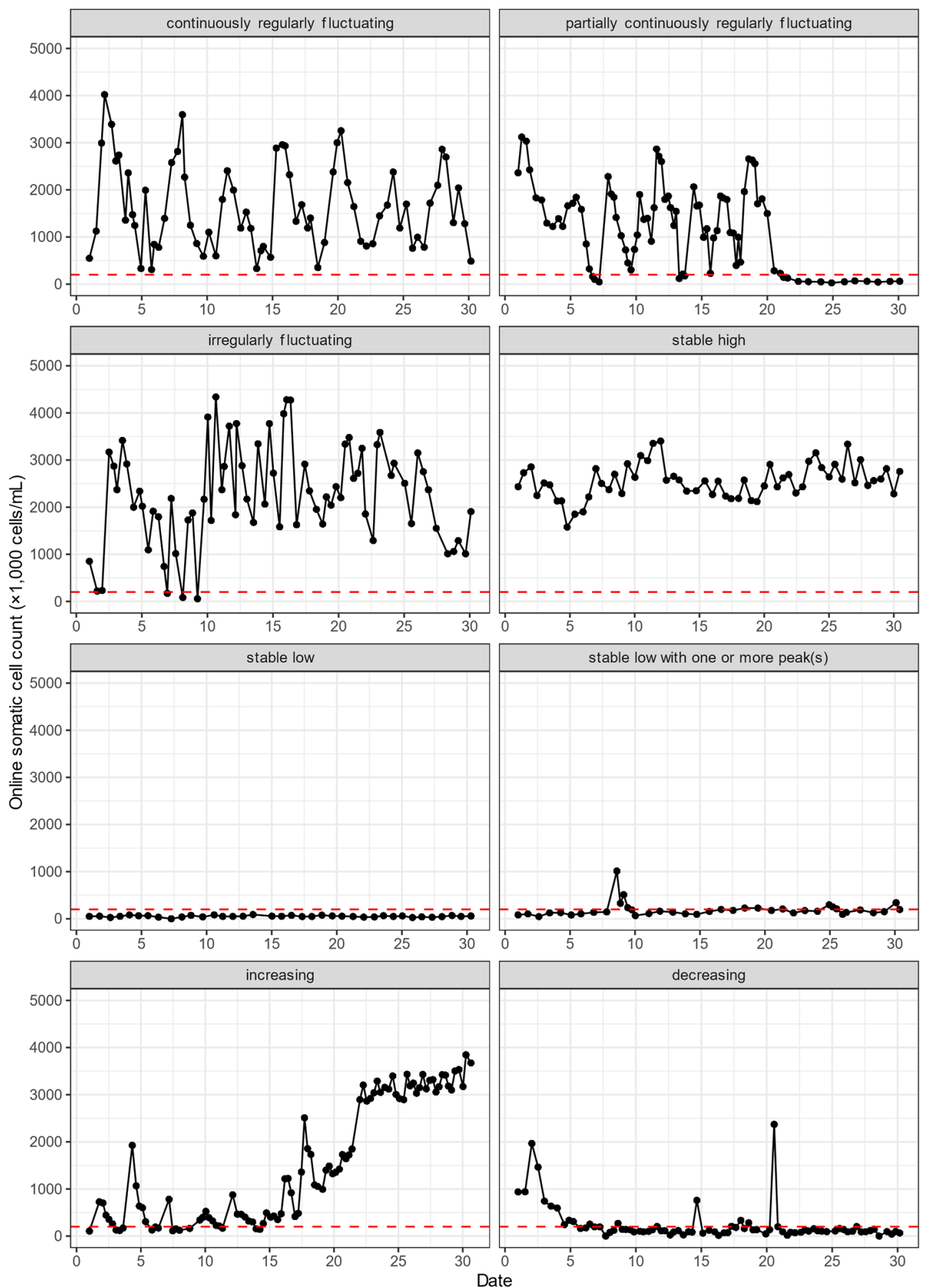

Figure 1. Representative examples of 30-d episodes of online SCC estimated by automated California Mastitis Test for each pattern category. The black dots connected by lines represent the online SCC, and the dashed horizontal line represents 200,000 cells $/ \mathrm{mL}$. 


\section{Factors Associated with rfSCC Pattern}

Data set 2 consisted of data from 1 group of cows in a Dutch AMS farm that were milked with an AMS equipped with an O-CMT sensor. This group of cows was prospectively studied from October 10, 2017, to February 27, 2018. Fresh cows entered the group and cows were dried off or culled, resulting in a constant group size of around 60 cows during the study period. Every 2 wk, milk samples were collected from all quarters of all cows present as follows: the udder was cleaned with a paper towel and the first 2 to 3 squirts of milk were discarded in a plastic cup to check for clots and other abnormalities, followed by disinfection of teat ends using cotton wool soaked in $70 \%$ alcohol. Then, a milk sample of 1 to $2 \mathrm{~mL}$ was collected in a plastic sterile vial that was stored on melting ice and transported to the laboratory to be cultured within the same day. Bacteriological culturing was performed according to National Mastitis Council guidelines (NMC, 2017), and isolates were identified using MALDI-TOF (Bizzini and Greub, 2010). In short, $10 \mu \mathrm{L}$ of quarter milk was inoculated on the sheep blood agar plate at $37^{\circ} \mathrm{C}$, and results were read at $24 \mathrm{~h}$ and $48 \mathrm{~h}$. Samples with $>2$ morphologically different colonies were defined as contaminated samples, according to the NMC (2017) guideline. In that case, colonies were not subjected to MALDI-TOF identification.

At every milking during the study period, the OCMT in this group of cows was measured. This data of each cow was displayed as a graph similar to the graphs mentioned above, but with the $\mathrm{x}$-axis spanning the entire period that the cow was part of the study. These graphs were judged by the 4 raters to identify episodes in which the rfSCC pattern was present. The raters were asked to record the start and end date(s) of the fluctuating pattern(s) for every graph. Next, in a collective meeting, discrepancies were discussed, resulting in consensus on the periods where the rfSCC pattern was present. Based on the quarter-milk sample culture results, pathogen species found in the rfSCC patterns were described.

To identify factors associated with the rfSCC pattern, we performed a logistic regression with a random herd effect, using the rfSCC with pattern 1 and 2 (yes or no) as the dependent variable, and parity (primiparous versus multiparous), average milking interval within rfSCC episode, DIM at the beginning of rfSCC pattern (modeled as a categorical variable with the 4 categories 1-40, 41-100, 101-200, 200-305), and season (summer, autumn, winter, spring) of the rfSCC pattern as independent variables using the glmer function in the lme4 package version 1.1-25 (Bates et al., 2015) in $\mathrm{R}$. The variance components of the logistic regression were extracted by using get_variance function in insight package version 0.13.1 (Lüdecke et al., 2019). The proportion of variance explained by the random herd effect was calculated as the variance explained in random effect divided by the total variance (variance explained by fixed effect + variance explained by random effect + residual variance). Model selection was performed using Akaike's information criterion.

\section{RESULTS}

\section{Prevalence of rfSCC}

In total, 669,170 records from 2,762 cows in 55 herds from 6 countries (Canada, Denmark, Germany, Finland, France, and Norway) were included in data set 1. The overall herd geometric mean SCC across all herds was $147 \times 1,000$ cells $/ \mathrm{mL}(2.5-97.5 \%$ quantile: 75 to $223 \times 1,000$ cells $/ \mathrm{mL}$ ). Detailed descriptive statistics of the entire data set are provided in Table 1.

The numbers of episodes within each category classified by each of the 4 raters and the consensus about categories 1 and 2 (the rfSCC categories) after discussion are provided in Table 2. The estimated consensus proportion of pattern 1 (continuously regularly fluctuating) was $2.2 \%$ (22/1,000 episodes in 16 herds in 5 coun-

Table 1. Numbers of farms, cows, and records per country in data set 1 and farm average milk yield $(\mathrm{kg})$ and $\mathrm{SCC}(\times 1,000 \mathrm{cells} / \mathrm{mL})$ estimated by the online California Mastitis Test (O-CMT) within the time period from January 1, 2015, to April 29, 2016

\begin{tabular}{lccccc}
\hline Country & $\begin{array}{c}\text { Total } \\
\text { number } \\
\text { of farms }\end{array}$ & $\begin{array}{c}\text { Median number } \\
\text { of cows per farm } \\
(2.5-97.5 \% \text { quantile })\end{array}$ & $\begin{array}{c}\text { Median number } \\
\text { of records } \\
(2.5-97.5 \% \text { quantile })\end{array}$ & $\begin{array}{c}\text { Farm average } \\
\text { daily milk } \\
\text { yield per cow } \\
(2.5-97.5 \% \text { quantile })\end{array}$ & $\begin{array}{c}\text { Farm geometric } \\
\text { mean of O-CMT } \\
(2.5-97.5 \% \text { quantile })\end{array}$ \\
\hline 1 & 1 & 78 & 35,858 & 28 & 179 \\
2 & 21 & $196(69-1,032)$ & $231,969(53,612-865,168)$ & $32(26.7-35.9)$ & $175(84-215)$ \\
3 & 9 & $294(158-493)$ & $198,544(108,995-296,149)$ & $35.3(27.7-41.5)$ & $162(137-203)$ \\
4 & 1 & 78 & 59,297 & 33.4 & 102 \\
5 & 13 & $100(75-197)$ & $82,060(43,550-141,393)$ & $30.8(26.4-34.4)$ & $120(75-196)$ \\
6 & 10 & $65(37-92)$ & $40,998(26,779-53,734)$ & $29.9(27-33.3)$ & $151(111-235)$ \\
Total & 55 & $138(45-789)$ & $98,357(34,587-682,643)$ & $31.8(26.5-39.0)$ & $147(75-223)$ \\
\hline
\end{tabular}




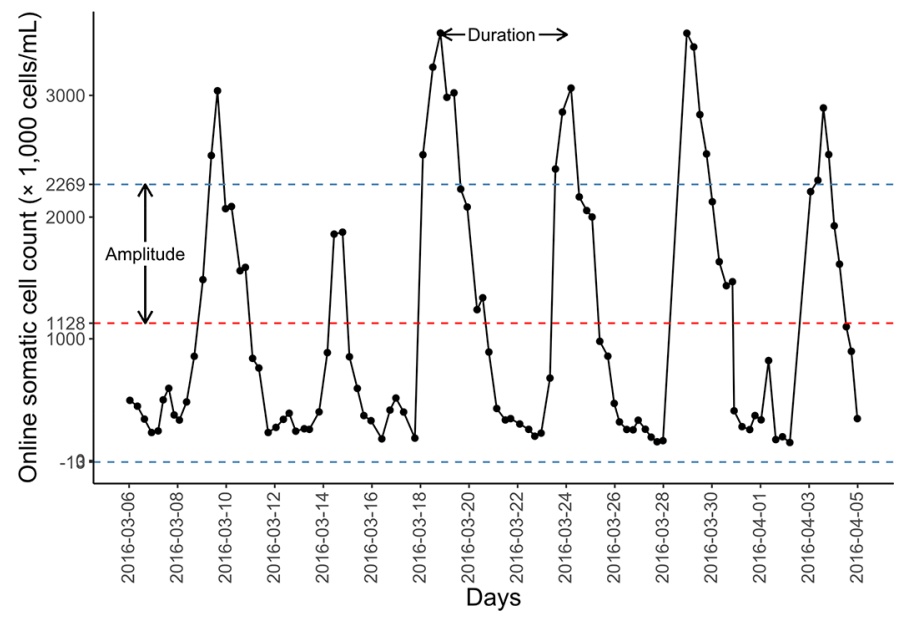

Figure 2. Example of a fluctuating SCC (estimated by automated California Mastitis Test) pattern to illustrate how amplitude (the distance between the middle and the outer dashed lines), the minimum and maximum of SCC, and duration of the pattern were estimated by spectral analysis.

tries) and pattern 2 (partially continuously regularly fluctuating) was $2.5 \%$ (25/1,000 episodes in 14 herds in 5 countries). Therefore, the total proportion of episodes displaying the rfSCC pattern (including both pattern 1 and 2 ) was estimated at $4.7 \%$ (95\% CI: $3.5-6.2 \%)$. The average SCC of the $22 \mathrm{rfSCC}$ episodes was $844 \times 1,000$ cells $/ \mathrm{mL}(2.5-97.5 \%$ quantile: 405 to $151 \times 1,000$ cells/ $\mathrm{mL})$. The median contribution per cow with the rfSCC pattern to the daily herd SCC was 1.8\% (2.5-97.5\% quantile: $0.1 \%$ to $22.4 \%$ ). According to the conservative definition, a proportion of $1.5 \%$ was classified as rfSCC; according to the most liberal definition, a proportion of $10.6 \%$ of all episodes was classified as rfSCC. The overall interrater agreement on 8 categories, according to Fleiss' kappa, was estimated to be 0.74 , which was mainly driven by the high agreement between raters on the stable low pattern. Raters had moderate agreement on pattern 1 specifically, but poor agreement on most other patterns, except the "stable low pattern" (Table 2).

\section{Characterization of rfSCC}

The amplitude and duration of rfSCC pattern are illustrated in Figure 2. All 22 episodes of data set 1 that were collectively identified as pattern 1 are displayed in Figure 3. Based on these patterns, the median of the average SCC of episodes was estimated as $701 \times 1,000$ cells/mL (2.5-97.5\% quantile: $539-1,162 \times 1,000$ cells/ $\mathrm{mL}$ ), the median duration of the fluctuating cycles was estimated at $4.1 \mathrm{~d}(2.5-97.5 \%$ quantile: $3.7-4.9$ d), and the median amplitude at $552 \times 1,000$ cells/ $\mathrm{mL}(2.5-97.5 \%$ quantile: $409-886 \times 1,000$ cells $/ \mathrm{mL})$, as displayed in Figure 3. The SCC recordings for all cows that displayed the rfSCC pattern in a 30-d episode are provided in Supplemental Figures S1 to S22 (https:/ /data.mendeley.com/datasets/rz69vxxm99/1; Deng, 2021). These figures show that the fluctuating pattern generally lasted for much longer than $30 \mathrm{~d}$.

\section{Factors Associated with rfSCC}

In data set 2 , the rfSCC pattern was found in 6 out of 81 cows that were monitored from September 26, 2017, to February 27, 2018. The SCC measurements over time in combination with the culture results of these cows are shown in Supplemental Figures S23 to S28 (https: //data.mendeley.com/datasets/rz69vxxm99/1; Deng, 2021). In several cows, one of the quarters was persis-

Table 2. Classification of 1,000 30-d episodes from individual cows in 55 dairy herds from 6 countries with highly frequent SCC measurements ${ }^{1}$

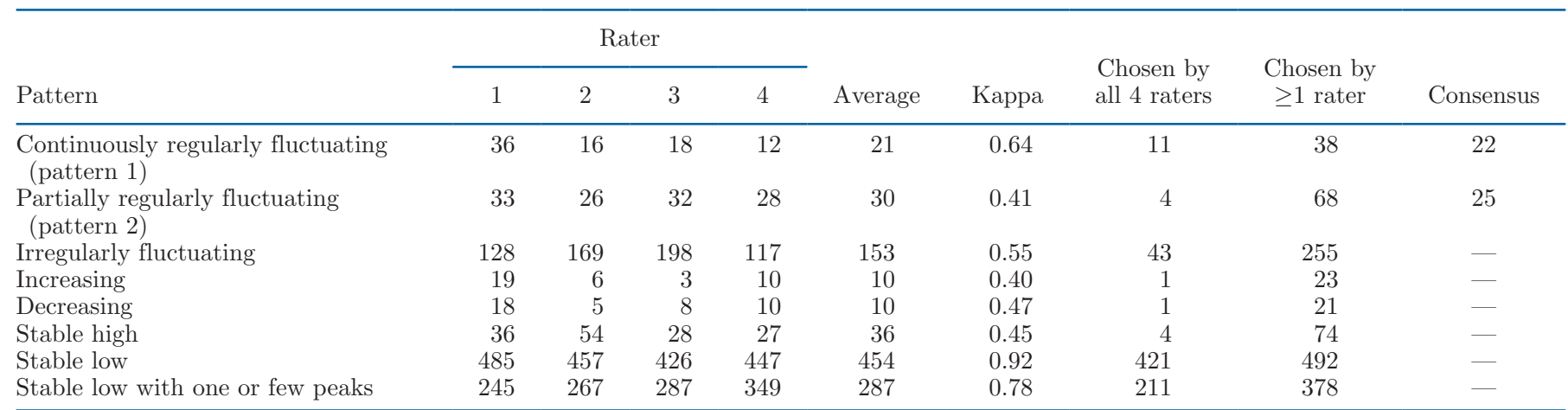

${ }^{1}$ Each episode consists of SCC values measured by a sensor system based on the California Mastitis Test. The episodes were classified into 8 pattern categories. The categorization of 4 different raters is presented, as are the average and Fleiss' kappa, indicating the interrater agreement, the number of episodes chosen by at least 1 of the raters, all the raters collectively, and the consensus after discussion (only for the first and the second pattern). 

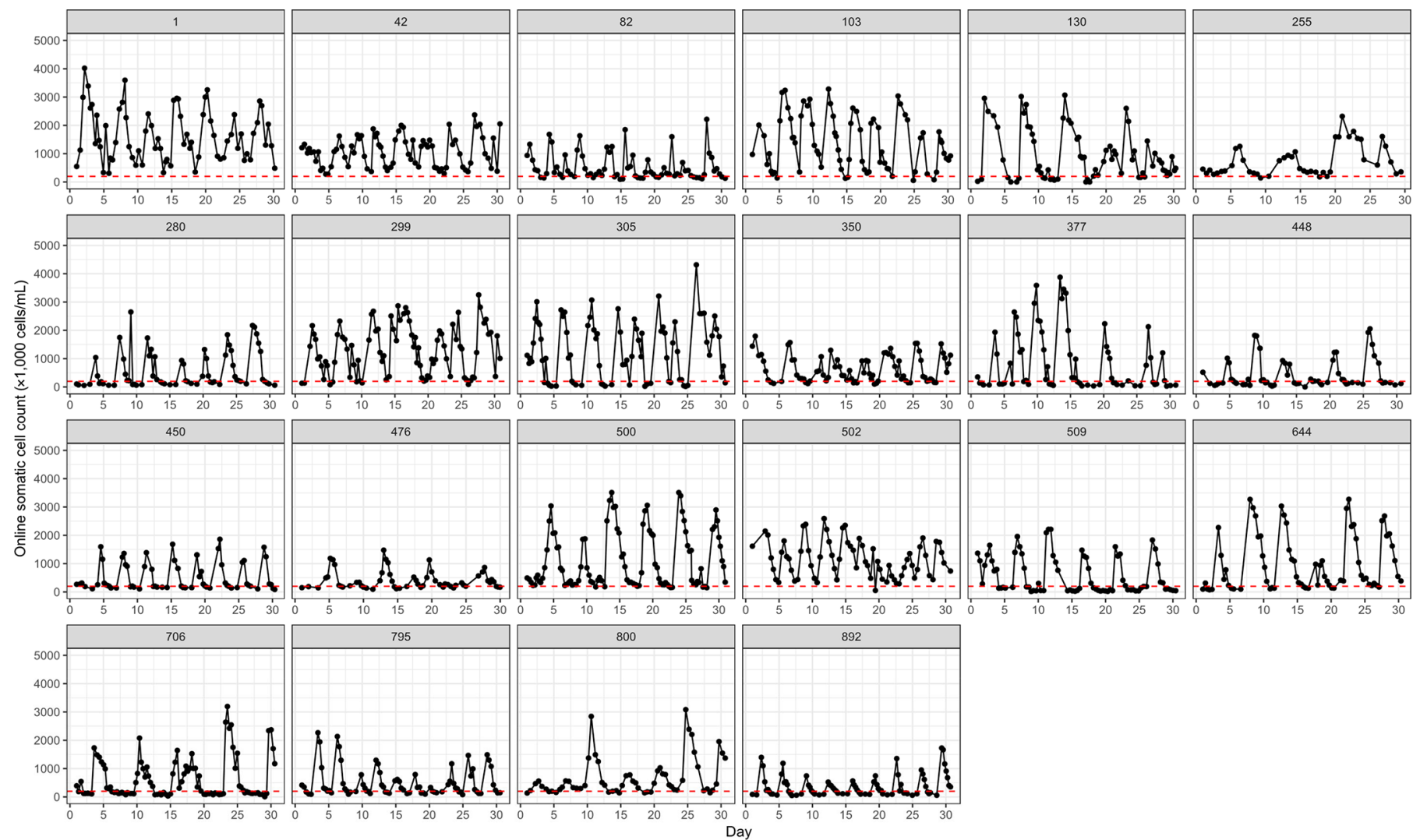

Figure 3. All 22 SCC episodes that were categorized as continuously regularly fluctuating by all 4 independent raters out of 1,000 episodes that were visually classified into 8 categories of SCC patterns. The dashed horizontal line represents 200,000 cells $/ \mathrm{mL}$.

tently infected as follows: cow 1 had a chronic infection in the right rear quarter with Staphylococcus haemolyticus, cow 2 had a chronic infection with Staphylococcus aureus in the right front quarter, cow 3 had a chronic infection in the right rear quarter with Enterococcus species, and cow 4 had a chronic infection in the left rear quarter with Streptococcus dysgalactiae and with Corynebacterium spp. in the right rear quarter. We did not find noticeable differences in quarter-milk culture results between cows with and without rfSCC patterns. These figures show that a variety of pathogens was cultured from various quarters of the cows with the rfSCC pattern, but gave no indication that a certain pathogen species or group was associated with this SCC pattern.

The results from the logistic regression model showed that the occurrence of the rfSCC pattern was not significantly associated with average milking interval, DIM, or season but was significantly positively associated with parity (odds ratio multiparous vs. primiparous $=$ 4.95; $P=0.0113 ; 95 \%$ CI:1.65-21.6). The herd identification was excluded from the final model as fixed effect, and the proportion of total variance attributed to the random herd effect was $11.7 \%$.

\section{DISCUSSION}

This study aimed to estimate the proportion of the newly described rfSCC pattern to characterize it and to describe the pathogens found in quarters from cows showing the rfSCC pattern. To the best of our knowledge, this is the first report describing such a SCC pattern. This was based on data from a large number of dairy herds and cows with highly frequent SCC measurements. Previous studies that described SCC patterns were mostly executed in a small number of herds with relatively frequent measures of SCC (e.g., Sears et al., 1990; Schukken et al., 2003; Urioste et al., 2010) or in large numbers of herds with infrequently measured SCC (e.g., de Haas et al., 2004). Because of the setup of those studies, none of them were able to report the rfSCC pattern as we have defined it. It is the application of the automated, online SCC sensors (in our case O-CMT) that enables a close monitoring of individual cow udder health for a longer time in a large number of animals.

The frequently measured O-CMT enables the study of SCC dynamics on a real time basis, whereas when 
SCC is measured in DHI programs, an SCC record is provided generally every 4 to 6 wk. Substantially more information on the dynamics of SCC could be identified by using the frequent O-CMT measurements and, for instance, changes in SCC that warrant intervention could be detected that would have gone unnoticed in normal DHI testing (Sørensen et al., 2016; Deng et al., 2020; Kirkeby et al., 2020).

The value of O-CMT measurement is an estimation of SCC based on the CMT principle rather than an exact measurement of SCC. Although the precision of the O-CMT measurements is not perfect, as indicated by Whyte et al. (2004), it can be used to monitor individual SCC dynamics as it is strongly associated with SCC, with a better accuracy in higher ranges of SCC (Deng et al., 2020). In cows with rfSCC patterns, the average SCC was generally high, and thus we considered the performance of O-CMT measurement sufficient for classifying rfSCC patterns. The repeatability of the OCMT measurements as indicated by intraclass correlation coefficient was 0.58 and was considered acceptable by Deng et al. (2020), although the O-CMT measurements may have resulted in a slight underestimation of the prevalence of the rfSCC pattern.

About $4.7 \%$ of the episodes (observed in 16 of the 55 herds) showed the rfSCC pattern. Although we could not find a clear association of the displayed rfSCC pattern with a specific pathogen species in the cows in data set 2, most of the cows with the rfSCC pattern did have positive culture results in one or more quarters. Although our results do not suggest that a single pathogen species is associated with the rfSCC pattern, the observations in this herd suggest that the rfSCC pattern is an indication of subclinical mastitis. However, future work is needed to identify if this is true for other herds. If indeed rfSCC indicates subclinical mastitis, a proportion of $4.7 \%$ is substantial, given that the overall prevalence of subclinical mastitis at the cow level has been estimated at $15.8 \%$ on Dutch dairy farms (Santman-Berends et al., 2016). In addition, rfSCC cows showed the pattern persistently; therefore, they may contribute substantially to a higher bulk tank SCC. It is also possible that the rfSCC is a physiological phenomenon, not actually representing disease (e.g. linked to fluctuations in milk yield or milking interval). Physiological variation has been described to account for a large proportion of the variation of composite milk SCC within individual dairy cows (Nørstebø et al., 2019; Deng et al., 2020).

The agreement between the 4 raters on the 8 different SCC pattern categories varied. The kappa value for the SCC pattern categories "partially regularly fluctuating," "irregularly fluctuating," "increasing," "decreasing," and "stable high" were in the range 0.40 to 0.55 , which is poor. An explanation for these low agreements could be that these SCC patterns were not as clearly distinct from the other patterns as the rfSCC pattern or the "stable low" pattern. We noticed that rater 1 classified substantially more episodes as rfSCC than the other raters. Clearly, this rater had a less stringent concept of the pattern in mind, despite the discussions with all 4 raters that had preceded the rating process. Given the fact that the consensus resulted in a higher number of rfSCC episodes than what was determined by either of the 3 other raters, it seems that rater 1 had a lower specificity but a higher sensitivity to identify the rfSCC pattern.

The geometric mean of the average SCC within rfSCC episodes $(774 \times 1,000$ cells $/ \mathrm{mL} ; 2.5-97.5 \%$ quantile: $405-1,515 \times 1,000$ cells $/ \mathrm{mL}$ ) was higher than the geometric mean SCC of chronic IMI caused by non-aureus Staphylococcus IMI (Bexiga et al., 2014), but lower than the median SCC of chronic IMI caused by Escherichia coli (Döpfer et al., 1999). This shows that the increase in SCC when the rfSCC pattern is present is substantial. In addition, at the peaks of the fluctuations, SCC was often $>2,000 \times 1,000$ cells $/ \mathrm{mL}$, although the estimated amplitude of the rfSCC pattern varied substantially between cows. This may reflect differences in host response between cows, but may also be caused by differences in pathogen species leading to the rfSCC. As the spectral analysis used to estimate the amplitude of the rfSCC pattern fits a curve to the data, and thereby smooths the peaks, the actual amplitude of the rfSCC may have been slightly underestimated as illustrated by Figure 2 .

The median duration of cycles of around $4 \mathrm{~d}$ was shorter than the estimated duration of a short IMI caused by E. coli $(6.7 \mathrm{~d})$ or Staph. aureus ( $6.3 \mathrm{~d}$; Bannerman et al., 2004). The number of pathogens used to infect a mammary gland in an experimental setting, as performed by Bannerman et al. (2004), is often much higher than in natural infections in the field, resulting in a stronger inflammatory reaction and likely a shorter duration of the IMI compared with a natural infection. Therefore, and also because of the stability of the observed pattern, we considered it very unlikely that each peak represented a new IMI, and we assumed that the rfSCC patterns represented a continuation of the same IMI infection. This is supported by the fact that the rfSCC pattern generally was very persistent and lasted for much longer period than 1 mo (Supplemental Figures S1-S22 (https://data.mendeley.com/ datasets/rz69vxxm99/1; Deng, 2021). As our data were censored, we could not objectively quantify the length of the pattern. We observed that the duration of IMI $(\mathrm{SCC}>200,000$ cells $/ \mathrm{mL})$ in cows with rfSCC pattern was generally much longer than cows with IMI but did 
not display the rfSCC pattern. As mentioned before, we are not sure that the rfSCC is caused by IMI. However, as an increase in SCC mainly reflects the immune response of a cow to an invading pathogen (Schukken et al., 2011; Bruckmaier and Wellnitz, 2017), fluctuations in SCC may reflect fluctuations in pathogen load. Regular fluctuations of SCC may be the result of a system similar to the predator-prey cycle (Loor et al., 2011), where the somatic cells suppress the infection, which then diminishes, enabling the pathogen to grow again and elicit a new SCC response. A cyclic shedding pattern of Staph. aureus has been described in the past in an experimental setting (Sears et al., 1990). However, in a later observational study, this phenomenon was not described (Walker et al., 2011). In addition, the strong decrease in SCC that we saw seemed unlikely to happen within a few days after the stimulus of the bacteria had been removed or decreased (Schukken et al., 2003). We tested the association between parity, average milking interval, DIM, season, and the occurrence of the rfSCC pattern. Of these variables, only parity was significantly $(P=0.0113)$ and positively associated with $\mathrm{rfSCC}$ pattern. The rfSCC pattern only occurred in some of the herds (with a low average herd prevalence of 0.02 and a large range), but whether it was herd or cow specific warrants further studies. Possibly, the affected cows had an impaired immunity, but the underlying mechanism leading to the occurrence of the rfSCC pattern is still unclear, and future research to identify the determinants of rfSCC is needed. As we showed the rfSCC pattern to be persistent over long periods of time, this research can be done by identifying cows that display the pattern based on routinely-collected data, subjecting these animals and a randomly chosen set of control animals to repeated culturing of milk samples, and identifying potential associations between causative agents and quarter level SCC measurements to identify whether a single quarter or multiple quarters display the pattern. Also, the use of differential SCC may be used to identify if specific cell fractions are mainly fluctuating. In addition, risk-factor studies can attempt to study associations with a larger set of risk factors than what we had access to in our study based on routinely measured data. This should provide new clues to unravel the causal mechanism behind this intriguing phenomenon.

\section{CONCLUSIONS}

The availability of novel online sensor systems that allow for routine measurement of SCC on a very frequent (daily up to every milking) basis enables detailed research in SCC patterns. In this study, we found that about $4.7 \%$ of the cows showed a newly described rf-
SCC pattern, which was highly stable in nature and displayed a repeated pattern of substantial increase and decrease in SCC within approximately $4 \mathrm{~d}$. The mechanism behind rfSCC is unclear, and despite the fact that rfSCC was seen in a substantial proportion of the cows, the practical importance of this finding remains to be demonstrated.

\section{ACKNOWLEDGMENTS}

This project was partially sponsored with a personal grant to Z. Deng by the China Scholarship Council (Beijing, China; grant number: 201506350111). We greatly appreciate Lely Industries N.V. (Maassluis, the Netherlands) for providing the data, the farmer's participation in this study, as well as the help of the students in collecting milk samples. The authors have not stated any conflicts of interest.

\section{REFERENCES}

Bannerman, D., M. J. Paape, J. W. Lee, X. Zhao, J. C. Hope, and P. Rainard. 2004. Escherichia coli and Staphylococcus aureus elicit differential innate immune responses following intramammary infection. Clin. Diagn. Lab. Immunol. 11:463-472. https://doi.org/ 10.1128/CDLI.11.3.463-472.2004.

Bates, D., M. Mächler, B. Bolker, and S. Walker. 2015. Fitting linear mixed-effects models using lme4. J. Stat. Softw. 67:1-48. https:// doi.org/10.18637/jss.v067.i01.

Bexiga, R., M. G. Rato, A. Lemsaddek, T. Semedo-Lemsaddek, C. Carneiro, H. Pereira, D. J. Mellor, K. A. Ellis, and C. L. Vilela. 2014. Dynamics of bovine intramammary infections due to coagulase-negative staphylococci on four farms. J. Dairy Res. 81:208214. https://doi.org/10.1017/S0022029914000041.

Bizzini, A., and G. Greub. 2010. Matrix-assisted laser desorption ionization time-of-flight mass spectrometry, a revolution in clinical microbial identification. Clin. Microbiol. Infect. 16:1614-1619. https://doi.org/10.1111/j.1469-0691.2010.03311.x.

Bruckmaier, R. M., and O. Wellnitz. 2017. Triennial Lactation Symposium/BOLFA: Pathogen-specific immune response and changes in the blood-milk barrier of the bovine mammary gland. J. Anim. Sci. 95:5720-5728. https://doi.org/10.2527/jas2017.1845.

de Haas, Y., R. F. Veerkamp, H. W. Barkema, Y. T. Gröhn, and Y. H. Schukken. 2004. Associations between pathogen-specific cases of clinical mastitis and somatic cell count patterns. J. Dairy Sci. 87:95-105. https://doi.org/10.3168/jds.S0022-0302(04)73146-X.

Deng, Z. 2021. Supplementary file. Mendeley Data, V1. https://doi .org/10.17632/rz69vxxm99.1.

Deng, Z., H. Hogeveen, T. J. G. M. Lam, P. P. P. van der Tol, and G. Koop. 2020. Performance of online somatic cell count estimation in automatic milking systems. Front. Vet. Sci. 7:221. https://doi.org/ 10.3389 /fvets.2020.00221.

Döpfer, D., H. W. Barkema, T. J. G. M. Lam, Y. H. Schukken, and W. Gaastra. 1999. Recurrent clinical mastitis caused by Escherichia coli in dairy cows. J. Dairy Sci. 82:80-85. https://doi.org/10.3168/ jds.S0022-0302(99)75211-2.

Gamer, M. 2019. Various Coefficients of Interrater Reliability and Agreement. Accessed May 11, 2020. https://cran.r-project.org/ web/packages/irr/irr.pdf.

Kirkeby, C., N. Toft, D. Schwarz, M. Farre, S. Nielsen, L. Zervens, S. Hechinger, and T. Halasa. 2020. Differential somatic cell count as an additional indicator for intramammary infections in dairy cows. J. Dairy Sci. 103:1759-1775. https://doi.org/10.3168/jds .2019-16523. 
Loor, J. J., K. M. Moyes, and M. Bionaz. 2011. Functional adaptations of the transcriptome to mastitis-causing pathogens: The mammary gland and beyond. J. Mammary Gland Biol. Neoplasia 16:305-322. https://doi.org/10.1007/s10911-011-9232-2.

Lüdecke, D, P. Waggoner, and D. Makowski. 2019. insight: A unified interface to access information from model objects in R. J. Open Source Softw. 4:1412. https://doi.org/10.21105/joss.01412.

NMC (National Mastitis Council). 2017. Laboratory Handbook on Bovine Mastitis. Natl. Mastitis Counc.

Nørstebø, H., G. Dalen, A. Rachah, B. Heringstad, A. C. Whist, A. Nødtvedt, and O. Reksen. 2019. Factors associated with milkingto-milking variability in somatic cell counts from healthy cows in an automatic milking system. Prev. Vet. Med. 172:104786. https:/ /doi.org/10.1016/j.prevetmed.2019.104786.

Santman-Berends, I. M. G. A., J. M. Swinkels, T. J. G. M. Lam, J. Keurentjes, and G. van Schaik. 2016. Evaluation of udder health parameters and risk factors for clinical mastitis in Dutch dairy herds in the context of a restricted antimicrobial usage policy. J. Dairy Sci. 99:2930-2939. https://doi.org/10.3168/jds.2015-10398.

Schukken, Y. H., D. Wilson, F. Welcome, L. Garrison-Tikofsky, and R. N. Gonzalez. 2003. Monitoring udder health and milk quality using somatic cell counts. Vet. Res. 34:579-596. https://doi.org/10 .1051 /vetres:2003028.

Schukken, Y. H., J. Günther, J. Fitzpatrick, M. C. Fontaine, L. Goetze, O. Holst, J. Leigh, W. Petzl, H. Schuberth, A. Sipka, D. G. E. Smith, R. Quesnell, J. Watts, R. Yancey, H. Zerbe, A. Gurjar, R. N. Zadoks, and H. Seyfert. 2011. Host-response patterns of intramammary infections in dairy cows. Vet. Immunol. Immunopathol. 144:270-289. https://doi.org/10.1016/j.vetimm.2011.08.022.

Sears, P. M. B. S., B. S. Smith, P. B. English, P. S. Herer, and R. N. Gonzalez. 1990. Shedding pattern of Staphylococcus aureus from bovine intramammary infections. J. Dairy Sci. 73:2785-2789. https://doi.org/10.3168/jds.S0022-0302(90)78964-3.
Seilmayer, M. 2019. Common Methods of Spectral Data Analysis. Accessed May 10, 2020. https://cran.r-project.org/web/packages/ spectral/index.html.

Sørensen, L. P., M. Bjerring, and P. Løvendahl. 2016. Monitoring individual cow udder health in automated milking systems using online somatic cell counts. J. Dairy Sci. 99:608-620. https://doi .org/10.3168/jds.2014-8823.

Urioste, J. I., J. Franzén, and E. Strandberg. 2010. Phenotypic and genetic characterization of novel somatic cell count traits from weekly or monthly observations. J. Dairy Sci. 93:5930-5941. https: //doi.org/10.3168/jds.2010-3301.

Walker, J. B., P. J. Rajala-Schultz, W. L. Walker, J. L. Mathews, W. A. Gebreyes, and F. J. DeGraves. 2011. Variation in daily shedding patterns of Staphylococcus aureus in naturally occurring intramammary infections. J. Vet. Diagn. Invest. 23:1114-1122. https:// doi.org/10.1177/1040638711425587.

Whyte, D. S., R. G. Orchard, P. S. Cross, T. Frietsch, R. W. Claycomb, and G. A. Mein. 2004. An on-line somatic cell count sensor. Pages 235-240 in Automatic Milking: A Better Understanding. A. Meijering, H. Hogeveen, and C. J. A. M. de Koning, ed. Wageningen Academic Publishers.

\section{ORCIDS}

Z. Deng $\odot$ https://orcid.org/0000-0002-9297-7956

T. J. G. M. Lam ( $)$ https://orcid.org/0000-0003-4601-2229

H. Hogeveen (ํ) https://orcid.org/0000-0002-9443-1412

G. Koop ๑ https://orcid.org/0000-0002-8752-0193 